\title{
Revisión de casos operados con diagnóstico clínico de apéndicitis aguda en pacientes
}

\author{
Gilberto Rodríguez-Herrera ${ }^{1}$
}

Justificación y objetivo: La apendicitis aguda es la urgencia quirúrgica abdominal más frecuente de la infancia. El objetivo del presente estudio fue realizar un análisis médico quirúrgico sobre el grupo de edad más común, género, grado de apendicitis y tratamientos previos de los pacientes apendicectomizados en el Servicio de Cirugía Pediátrica del Hospital de San Carlos.

Método: Se incluyeron 120 pacientes de ambos sexos, apendicectomizados en el Servicio de Cirugía Pediátrica del Hospital de San Carlos, con edades comprendidas entre los 0 y 13 años, durante el período de enero de 1999 a agosto de 2001. La recolección de los datos fue llevada a cabo por el investigador, en una revisión exhaustiva de las historias clínicas y los expedientes.

Resultados: Entre los 120 pacientes operados, el grupo de edad más frecuente es de 10 a 13 años, el género al que mayormente se le realizó apendicectomía fue el masculino, el grado clínico patológico que predominó fue el segundo. Entre los tratamientos previos más utilizados están los analgésicos no opioides y los antinflamatorios no esteroidales. Los pacientes que recibieron tratamiento médico previo presentaron mayor grado apendicular clínico patológico durante la apendicectomía.

Conclusiones: El diagnóstico de apendicitis aguda es principalmente clínico. Su retraso y el inicio de tratamiento médico son factores importantes que repercuten en la evolución y en el incremento de la morbilidad y mortalidad del paciente pediátrico.

Descriptores: Apendicitis aguda, grado clínico patológico, tratamientos previos, apendicectomía, diagnóstico temprano, enfermedad de morera, dolor abdominal agudo.

Recibido: 12 de diciembre, 2002

Aceptado: 18 de marzo, 2003

La apendicitis aguda es el cuadro quirúrgico de urgencia abdominal más frecuente. Según las estadísticas mundiales, es la causa principal del abdomen agudo quirúrgico.$^{1-8}$

Es una de las entidades clínicas mejor reconocidas, pero una de las que mayores problemas diagnósticos puede suponer para el clínico, ${ }^{9}$ constituyendo un desafío único y a veces frustrante. ${ }^{10}$

Del 7 al 12\% de la población general padece apendicitis aguda en algún momento de su vida.

Es una enfermedad poco frecuente en menores de 7 años. Alcanza su mayor frecuencia en personas entre los 15 y los 25

Abreviaturas: AINES, antinflamatorios no esteroidales. HSC,Hospital San Carlos.

Asistente en medicina general, Servicio de Emergencias, Hospital de San Carlos.

Correspondencia: Gilberto Rodríguez Herrera. Servicio de Emergencias del HSC,Alajuela, Costa Rica. drgilbertorodriguez@ hotmail.com años. Menos del 2\% de las apendicitis pediátricas se presentan en lactantes y es extremadamente rara en neonatos ${ }^{1-6}$

Es una enfermedad más grave en lactantes y niños que en adultos, porque el índice de ruptura es más alto (excede el $50 \%$ en menores de 6 años 1), lo que a su vez origina mayor morbilidad y mortalidad. La precisión diagnóstica de apendicitis aguda en niños es mucho más baja que en adultos. A ello contribuye un cuadro clínico menos "típico", con fiebre alta y vómitos, también el aspecto de que los lactantes no pueden proporcionar una historia de la enfermedad actual, y la falla del médico de no considerarla por su relativa rareza en los muy pequeños. El retraso en su diagnóstico y factores como la delgadez relativa de la pared del apéndice en los pacientes pediátricos ${ }^{1}$, y el hecho de que el epiplón es más corto, por lo que no alcanza a obliterar y delimitar la zona de inflamación, contribuyen a que los niños sufran peritonitis generalizada y gangrena más precozmente. ${ }^{4}$

Con respecto a la clasificación de la apendicitis aguda, tratando de corresponder con los estadíos clínicos, diferentes autores han propuesto las siguientes:

62 AMC, Abril-Junio 2003, vol 45 (2) 
Ellis las divide en apendicitis aguda perforada y no perforada11; Caballero las clasifica en tres estados conocidos como: edematosa, supurativa y perforada12; Pera las identifica en: catarral, cuando predomina el fenómeno de hiperemia y congestión (grado I); flegmonosa, cuando aparecen erosiones de la mucosa con supuración y exudados fibrinopurulentos en la serosa (grado II); gang renosa, cuando hay necrosis de la pared (grado III) y perforada (grado IV) ${ }^{13}$.

El niño con dolor abdominal agudo representa aún un dilema de enorme dificultad en el diagnóstico, incluso para el clínico más astuto. Es esencial la evaluación temprana. Los únicos medios para disminuir la frecuencia creciente con que surge la perforación apendicular son la anamnesis detallada, la exploración física minuciosa y la consulta quirúrgica rápida en el niño con signos de irritación peritoneal. ${ }^{1-3}$.

En el Hospital San Carlos se operan cerca de 40 pacientes por año, con diagnóstico preoperatorio de apendicitis aguda en niños de 13 ó menos años, y se trasladan al Hospital Nacional de Niños, anualmente entre 20 y 25 niños, con el diagnóstico de dolor abdominal en estudio o sospechas de apendicitis aguda.

El propósito del estudio fue determinar el grupo de edad en que hubo más incidencia de apendicitis, el género más frecuente, determinar si existe relación entre los pacientes que han sido medicados antes del diagnóstico de apendicitis aguda y el grado clínico-patológico de la apéndice encontrada en la apendicectomía.

\section{Materiales y métodos}

La investigación aquí expuesta es cuantitativa y analítica,estableciéndose la comparación de variables entre el grupo y estudiando estas según se den naturalmente.

Durante un periodo de 32 meses (enero de 1999 a agosto de 2001) se estudió en forma retrospectiva todos los pacientes menores o iguales a 13 años que fueron apendicectomizados. Los datos fueron obtenidos por el autor de los expedientes clínicos de cada paciente, en el Departamento de archivo del Hospital San Carlos.

Se elaboró un protocolo en donde se anotaron el número de expediente, la edad y el género. Los pacientes fueron divididos en cuatro grupos, según el grado clínico-patológico de la apéndice extraída, propuesta por Pera13. Se incluyó también en el protocolo el dato de si estos pacientes recibieron medicación previa a la consulta con un médico y el tipo de esta en los casos positivos.

Se ordenaron los tratamientos utilizados en el preoperatorio en ocho grupos de la siguiente manera: analgésicos, antiespasmódicos, antinflamatorios no esteroidales, antibióticos, catárticos, medicina natural, masaje abdominal o "sobado", y medicamentos o maniobras combinados, tales como: inyectable y pastilla, Alka Seltzer®, "sobado", analgésicos, antibióticos, antiácidos, desparasitantes, purgantes.
Los niños fueron divididos en cuatro grupos de edades: menores de 2 años, de 2 a 5 años, de 6 a 9 años y de 10 a 13 años.

Se revisó además el diagnóstico histopatológico emitido en el informe del Departamento de Patología.

Se relacionaron los resultados entre el grado clínico-patológico encontrado y el dato de si recibieron o no tratamiento previo los pacientes operados.

\section{Resultados}

De los 120 pacientes operados, 67 correspondían al sexo masculino $(55,83 \%)$ y 53 al femenino $(44,17 \%)$.

Del total de pacientes intervenidos, 93 tuvieron un diagnóstico histopatológico de apendicitis aguda supurada con periapendicitis, lo que representa el 77,5\%; 4 tuvieron diagnóstico patológico de apendicitis aguda supurada con periapendicitis con granuloma de Angiostrogilus costarricensis para un 3,33\%; 14 fueron considerados como apéndices sanas para un $11,66 \%$, y en 9 no había diagnóstico patológico, para un $7,5 \%$. Catorce pacientes fueron clasificados con diagnóstico clínico postquirúrgico de apendicitis aguda, por los cirujanos tratantes, a pesar de tener sus apéndices sanos por diagnóstico patológico. De las apéndices "sanas" 6 fueron en el sexo masculino $(42,85 \%)$ y 8 en el femenino $(57,15 \%)$.

Edad: Los pacientes se agruparon así:

a) Se operaron 3 pacientes menores de 2 años, de los cuales 1 tenía una apendicitis grado III, otra una apendicitis grado IV y la otra una apéndice sana.

b) De edades comprendidas de los 2 a los 5 años, se operaron 25 pacientes, 1 tenía apendicitis grado I (4\%); 10 de grado II (40\%); 6, grado III (24\%); 4, grado IV (16\%) y 4, apéndices sanas $(16 \%)$.

c) De los 6 a los 9 años se operaron 24 pacientes, de los cuales 5 eran apendicitis grado I $(20,83 \%) ; 14$, grado II $(58,33 \%)$; 3 grado III $(12,5 \%)$; 0 , grado IV y 2 tenían apéndices sanas $(8,33 \%)$.

d) De los 10 a los 13 años se operaron 68 pacientes, de los cuales 3 tenían apendicitis grado I $(4,41 \%) ; 31$, grado II $(45,58 \%) ; 13$, grado III $(19,11 \%) ; 7$, grado IV $(10,29 \%)$; 7 tenían apéndices "sanas" $(10,29 \%)$ y 7 apéndices no clasificadas (10,29\%) (Figura 1).

Por lo tanto, en total, de las 120 apendicetomías, 9 eran de grado I $(7,5 \%) ; 55$, de grado II $(45,83 \%) ; 23$, de grado III $(19,16 \%) ; 12$, de grado IV $(10 \%) ; 14$, apéndices sanas $(11,66 \%)$ y 7 no fueron clasificadas, a pesar de tener diagnóstico patológico de apendicitis (5,83\%) (Figura 2).

Tratamiento previo:

De los 120 pacientes operados, 38 recibieron tratamiento previo $(31,66 \%)$, que se clasificó de la siguiente manera, en orden descendente: 
Pacientes apendicectomizados divididos en grupos de edad, en la Región Huertar Norte

Enero de 1999 a agosto de 2001

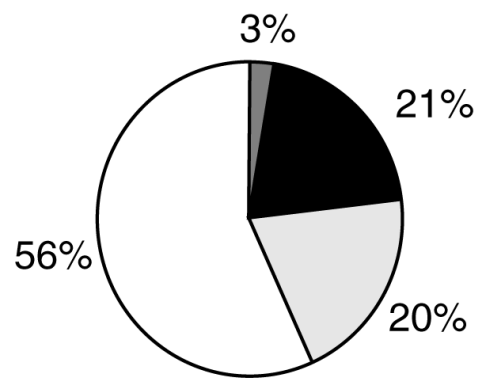

$<2$ años

2-5 años

6-9 años

10-13 años

Figura 1. La apendicitis aguda predominó en los pacientes con edades comprendidas entre los $10 y$ lo 13 años. En las edades comprendidas entre 2 y 5 años y 6 y 9 años existe una gran similitud que oscila entre el $20 \%$, respectivamente. La apendicitis en menores de 2 años es relativamente rara, con un $2,5 \%$.

- Tratamientos combinados: $12(31,77 \%)$

- Analgésicos: $11(28,94 \%)$

- "Sobados": $7(18,42 \%)$

- $\quad$ Antiespasmódico: 3 (7,89\%)

- Antinflamatorios no esteroidales: $2(5,26 \%)$

- $\quad$ Antibióticos: $1(2,63 \%)$

- Catártico: $1(2,63 \%)$

- Medicina natural: 1 (2,63\%) (Figura 3)

En los 38 pacientes que recibieron tratamiento previo al diagnóstico, se encontró la siguiente clasificación patológica de la apéndice extraída :

1 apendicitis grado I $(2,63 \%), 16$ apendicitis grado II $(42,1 \%), 11$ apendicitis grado III $(28,94 \%), 7$ apendicitis grado IV $(18,42 \%)$ y 3 apéndices sanas $(7,89 \%)$.

En los 82 pacientes que no recibieron tratamiento previo, la apendicitis se clasificó de la siguiente manera:

8 apendicitis de grado I $(9,75 \%), 40$ apendicitis de grado II (48,78\%), 12 apendicitis de grado III (14,63\%), 3 apendicitis de grado IV $(3,65 \%), 11$ apéndices sanas $(13,41 \%)$ y 9 apendicitis no clasificadas $(9,75 \%)$.

\section{Discusión}

El porcentaje de pacientes operados con diagnóstico clínico de apendicitis aguda que finalmente no fueron confirmados como tales en el estudio histológico (apéndices sanos), fue muy similar al referido en las diferentes series internaciona-
Grados de apendicitis, segúnclasificación clínico-patológica,

Región Huertar Norte

Enero de 1999 a agosto de 2001

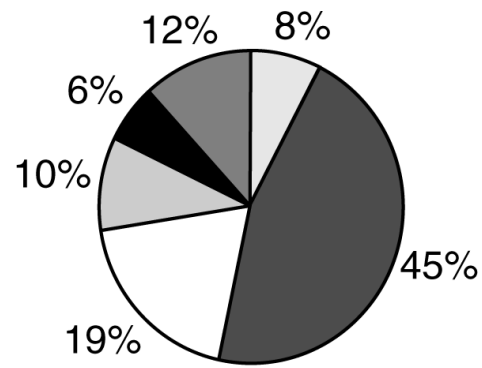

$\square$ I $\square$ II $\square$ III $\square$ IV $\square$ No clasificado $\square$ Sanas

Figura 2: Nótese un predominio de la apéndice grado II, en casi la mitad de los casos. El porcentaje de apéndices que se operaron a pesar de ser sanos, es de casi un $12 \%$. Alrededor de una tercera parte de las apendicectomías realizadas se clasificaron como grados III O IV.
Porcentaje de pacientes que recibieron tratamiento previo a la apendicectomía en la Región Huertar Norte Enero de 1999 a agosto de 2001

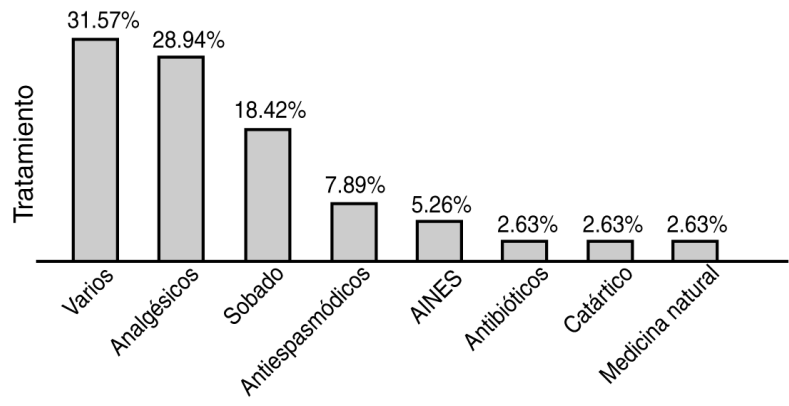

Figura 3.

les $(11,66 \%)$. Las revisiones consultadas por el autor mencionan como rango aceptable de un $10 \%$ a un $30 \%$ de apéndices saludables. Mc Burney razonó que la práctica de un procedimiento quirúrgico explorador rápido para asegurar la extirpación del apéndice antes de su ruptura, era mucho más segura que retardar el tratamiento quirúrgico con la esperanza de una curación espontánea ${ }^{1}$. Se considera que las moles tias y riesgos asociados a la laparotomía exploratoria, en el caso de "ausencia de enfermedad", son muy inferiores a la morbilidad y mortalidad (alrededor del $2 \%$ ) asociada con la perforación del apéndice y, además, relacionan estos resultados con la falta de signos y síntomas específicos y de sensibilidad y especificidad de los estudios de laboratorio ${ }^{4-9-9-14}$. Según este concepto, la apendicectomía de apéndices sanos representa el costo por pagar para minimizar el número de apendicitis agudas evolucionadas operadas y así disminuir la morbimortalidad ${ }^{7-9-15}$. Otros autores son más exigentes y consideran como rangos aceptables del $10 \%$ al $15 \%$ de apéndices 
sanas, pues consideran que la extirpación de un apéndice saludable podría ser perjudicial, al requerir un internamiento más prolongado y presentar una mayor tasa de efectos secundarios ${ }^{1-15-16}$.

Se refiere que la precisión de diagnóstico preoperatorio debe ser del $85 \%$, pues si es menor, probablemente se practiquen algunas operaciones innecesarias y procede un diagnóstico diferencial más riguroso. Por otra parte, una precisión siempre mayor del $90 \%$ también debe preocupar, ya que ello puede indicar que algunos pacientes con apendicitis aguda atípica se dejan en "observación", cuando deberían operarse con rapide $z^{4}$. Las apendicectomías por apéndice "sana"mostraron una diferencia entre el sexo masculino $(42,85 \%)$ y el femenino $(57,15 \%)$, lo que confirman diferentes estudios, en los cuales, al incluir la patología ginecológica, se aumentan los posibles diagnósticos diferenciales del dolor abdominal y, por lo tanto, las posibilidades de equivocar el diagnóstico.

Con respecto al género de los pacientes apendicectomizados, casi se equiparan, pero existe cierto predominio del género masculino. La bibliografía nos indica de un $60 \%$ de predominio masculino ${ }^{17}$.

Es muy interesante el comportamiento de las apendicitis agudas en los diferentes grupos de edad. En menores de dos años, la frecuencia en el estudio es baja (2,5\%), como se reporta, y se describe como entidad extremadamente rara ${ }^{2-17}$, e inclusive se señalan de cifras menores al $2 \%$ en lactantes y recién nacidos ${ }^{1}$. También se confirma lo difícil del diagnóstico temprano, lo que conlleva a aumentar las complicaciones. Se citan en la bibliografía cifras promedio de perforación apendicular, en menores de 6 años, de más del $50 \%$ y en preescolares del 50 al $85 \%$. $^{1-4-6}$

Los grados apendiculares I y II tienden a aumentar conforme la edad del niño lo hace, mientras que los grados III y IV y las apéndices sanos tienden a disminuir conforme aumenta la edad. Se podría concluir que el grado de apendicitis es inversamente proporcional a la edad del niño (Figura 4).

Llama la atención que algunos autores consideran que el bajo nivel socioeconómico está íntimamente relacionado con el incremento de las perforaciones apendiculares. Este varía del $15 \%$ en hospitales privados, al $25 \%$ o $30 \%$ en hospitales de caridad. ${ }^{4}$

Otro dato interesante es que, según trabajos analizados, al menos el $50 \%$ de las perforaciones ocurren antes de que el paciente se contacte con un centro de salud, y de un $65 \%$ a un $68 \%$ de todas las perforaciones, suceden antes de que el paciente sea evaluado por un cirujano.

En los $38(31,66 \%)$ pacientes que recibieron tratamiento previo al diagnóstico de apendicitis aguda, existió un predominio del uso de tratamientos combinados o analgésicos solos. Un estudio realizado en Colombia reporta que un $40 \%$ de los pacientes con diagnóstico de apendicitis aguda habían recibi-

\section{Grado de apendicitis aguda, en relación con el grupo de edad, la Región Huertar Norte Enero de 1999 a agosto de 2001}

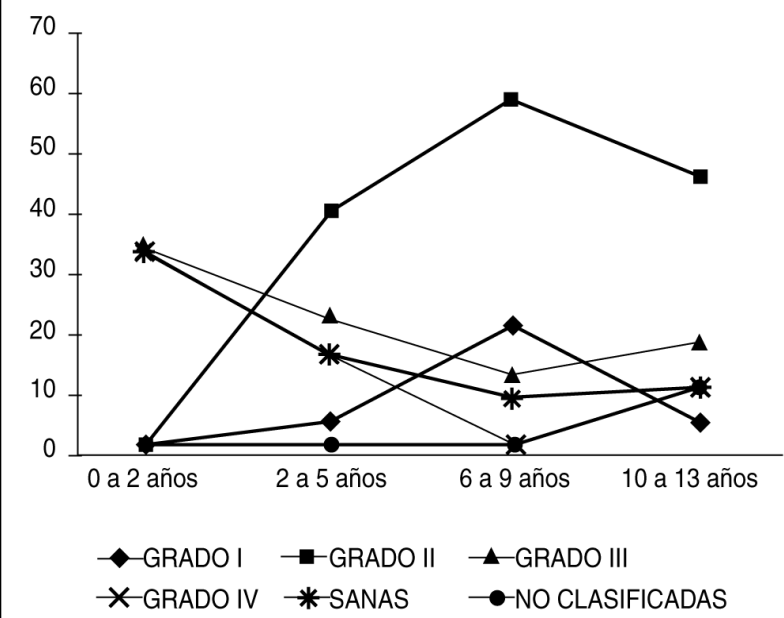

Figura 4: Esta figura indica los diferentes grados clínicos patológicos relacionados con los grupos etarios. En los niños menores de 2 años existe un predominio de las apéndices grados III y IV y las sanas. Conforme va aumentando la edad, se nota un declive en los grados apendiculares y un aumento de las apendicitis grado I y II y las no clasificadas.

\section{Comparación de apéndices extraidas en pacientes que recibieron o no tratamiento previo en la Región Huertar Norte Enero de 1999 a agosto de 2001}

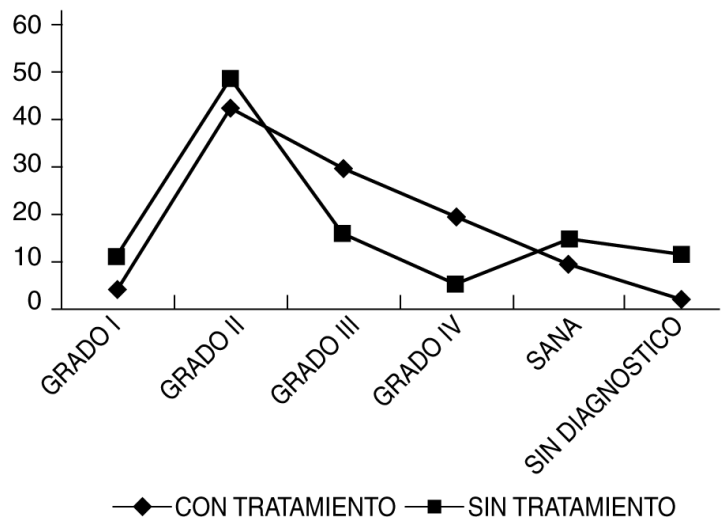

Figura 5: En los pacientes que recibieron tratamiento previo a la atención médica-quirúrgica existe un mayor porcentaje de apendicitis grados III y IV, mientras que en los que no recibieron tratamiento previo a la atención médica, predominan las grados I y II.

do tratamiento médico previo ${ }^{14}$. Resulta interesante que aún en estos tiempos de alta tecnología existan creencias tan primitivas como la comúnmente llamada "pega". A pesar de que el llamado "sobado" en el paciente no va a afectar la evolución real de la apendicitis aguda, sí va a atrasar la consulta médica temprana, esperando a ser "curados" luego del tratamiento tradicional, se trata de un factor importante por considerar dentro de la historia clínica en nuestra región ${ }^{18}$. 
El retardo en el diagnóstico y el inicio del tratamiento médico son elementos que repercuten en la evolución y en el incremento de la morbilidad y mortalidad ${ }^{19}$.

En el interrogatorio se debe anotar si el paciente recibió analgésicos, por que estos enmascaran el dolor. Si los recibió, siempre se deberá esperar hasta que pase el efecto para valorar el paciente de nuevo. También se debe indagar sobre el uso de antibióticos. El paciente con apendicitis aguda que recibió antibióticos pueden presentar pocos o ningún síntoma. Por esta razón, si se plantea la duda de que exista apendicitis, el paciente no debe recibir tratamiento antibiótico hasta descartar la duda. Es preferible esperar 12 ó más horas hasta que se aclare el cuadro ${ }^{20}$. Los enemas o laxantes pueden precipitar la perforación.

Varios autores demuestran que existe un predominio de apendicitis grados I y II en los pacientes que no recibieron tratamiento previo y una menor incidencia de este en pacientes con apendicitis grados III y IV, en comparación con los que recibieron algún tipo de medicación (Figura 5) ${ }^{20-21}$.

Los diagnósticos preoperatorios erróneos más comunes corresponden a más del 75\%, y en orden descendente son: linfadenitis mesentérica aguda, ausencia de trastorno patológico orgánico, afección inflamatoria pélvica aguda, torcimiento de un quiste ovárico o rotura de un folículo de Graff y gastroenteritis aguda. ${ }^{4}$

En la zona de la Región Huetar Norte y probablemente en muchos otros lugares de nuestro país, se debe incluir dentro de los diagnósticos preoperatorios la Enfermedad de Morera, producida por el Angiostrongilus costarricensis. Pues se ha encontrado que podría ser causante de apendicitis aguda al obstruir la luz intestinal, generalmente con un plastrón eosinofílico.

La tasa de mortalidad para la apendicitis en niños menores de 2 años es inferior al 0,1\%. En los lactantes del $10 \%$ y en los recién nacidos puede ser hasta del $80 \%{ }^{1-6}$.

La mortalidad operatoria general fluctúa entre el $0.2 \%$ y el $1 \%$ y habitualmente se concentra en pacientes que son intervenidos con perforación del apéndice, peritonitis y alguna otra condición agregada como edad avanzada, diabetes mellitus, obesidad mórbida u otras enfermedades concomitantes que hacen que el riesgo quirúrgico sea particularmente alto.

La morbilidad operatoria es del $0.83 \%$ para la apendicitis no perforada y del $6 \%$ para la apendicitis perforada; la infección de la herida quirúrgica es la complicación más frecuente, seguida por la infección de vías urinarias, neumonía, absceso residual y otras ${ }^{4}$. En el estudio no hay datos de que algún paciente halla fallecido. Las cifras de mortalidad en la bibliografía oscilan entre el $0,43 \%$ en niños mayores, hasta un $80 \%$ en recién nacidos. ${ }^{1-4}$

\section{Agradecimientos y colaboradores}

A la señora Laura Campos y su equipo en el área de registros médicos del HSC.

Al Dr. Dagoberto Alfaro Ávila, cirujano pediatra, por su intenso trabajo por intentar salvar la vida de muchos niños.

A la Dra. Catalina Sáenz, médico general del HSC, y a todas aquellas personas que brindaron su colaboración para concluir este estudio.

A mis padres, hermanos y a mi novia, que siempre me han apoyado.

\section{Abstract}

Justification and Objectives: Acute appendicitis is the most frequent abdominal surgical emergency in children. The objective of the present study was to conduct a medical surgical analysis regarding the most frequent age group, gender, grade of appendicitis and previous treatments used in appendicectomized patients at the Pediatric Surgery department of the San Carlos Hospital.

Methods: One hundred and twenty patients of both genders were appendectomized at the Pediatric Surgery department of the San Carlos Hospital, their ages ranged from hours old neonates to thirteen years old, between January 1999 to August 2001. The gathering of data was carried out by the author in an exhaustive review of clinical histories and files.

Results: Among the 120 patients operated, the most frequent age group was from 10 to 13 years old, there were more males than females; most were classified as being second grade appendicitis. Non steroidal anti-inflammatory drugs as well as non opiate analgesics were among the previous medical treatments more widely used. The patients who stated having received previous medical treatment presented a higher grade of clinical appendicitis during surgery and in the pathology specimens.

Conclusions: The diagnosis of acute appendicitis is mainly clinical. Late diagnosis and delay in medical treatment are important factors that increase the morbidity and mortality of the pediatric patient.

\section{Referencias}

1. Ashcraft K, Holder T, Cirugía pediátrica, Apendicitis, México: McGraw-Hill, Segunda edición, 1995, 39: 484-491.

2. Madrigal G, Manual de diagnóstico y terapéutica en pediatría, Apendicitis aguda, Primera edición, San José Costa Rica,Editorial de la Universidad de Costa Rica, 1995:92-102.

3. Hatch E,Clínicas Pediátricas de Norteamérica,Cuadro abdominal agudo en niños,USA: Emalsa S.A, 1985, 32: 1201-1213.

4. Schwartz S, Shires T, Spencer F, Principios de cirugía, apéndice, México: McGraw-Hill, Sexta edición,1995, Vol. II: 1347-1357.

5. Doherty G, Meko J,. Olson J, Peplinski G, Worrall N, Washington Manual de cirugía. Apendicitis aguda, Segunda edición. Marban Libros, Madrid España. 2001: 228-235. 
6. Hardin M, Acute appendicitis: review and update. Am Fam Physician 1999; 60: 2027-34

7. Santiago P, Barberousse C. Correlación clínico-histopatológica en apendicitis aguda. GEN 1995; 49(2): 140-4.

8. Talavera F, Prueba radiológica para la detección de casos dudosos de apendicitis aguda en niños. Am Surg 217:115, 1993.

9. Cotran R, Kumar V, Robbins S, Patología estructural y funcional. Apéndice. México: McGraw-Hill, Cuarta edición, 1990, Volumen II: 950-952.

10. Aach R, Ansell J, Atkins E, Bauwens D, Blacklow R, Cahill G, et al. Signos y síntomas de McBryde. Dolor abdominal. Prensa Técnica S.A, 1990:173-187.

11. Schwartz S, Ellis H,Cowles W, Maingot:Operaciones abdominales, 8 a Ed, Buenos Aires, Edit Médica Panamericana S.A.,1986: 1225-53.

12. Caballero F, Duarte L, Morales L: Apendicitis aguda. Trib Med 1991; 83(4):143-6.

13. Pera C,Cirugía:Fundamentos,indicaciones y opciones técnicas. 1a Ed, Barcelona,Ediciones Científicas y Técnicas S.A. 1983.

14. Matamoros M,Cortes J, Emergencias en pediatría. Instituto Costarricense de Investigaciones Clínicas, Primera edición, San José CR, Editorial ICIC 1996:134-141.
15. Sansevero A, J. appendicitis. Online Pediatric Surgery Handbook for Residents and Medical Students. URL: http://www.mja.com.au/> (C) 1998 Medical Journal of Australia.

16. Isaac Lobo. Estudio advierte sobre extirpación de apéndices. Periódico Al día . Costa Rica. Miércoles 17 de julio de 2002. Sección Sociedad. Página 2.

17. Sadovsky R, Acute appendicitis in children: emergency department diagnosis and management. Ann Emerg Med July 2001;36:39-51.

18. Dale R, Acupuntura con sus dedos: Un sistema curativo de dieciocho puntos Dialectc Publications, inc. Surfside, Florida, 1989

19. Suárez R, Blanco R, Sánchez P, Mier J, Castillo A, Robledo F, et al Apendicitis aguda complicada. El valor diagnóstico temprano. Cir Ciruj 1997; 65(3):65-68.

20. Echavarría R, Ferrada R, Kestenberg A. Apendicitis aguda. Urgencia quirúrgica. ASPROMEDICA y Universidad del Valle. Cali. 1995:. 6265

21. Scott H Plantz. EMedicine Consumer Journal,December 17,2000, Volume 1, Number 12 